\title{
Mechanical properties of WC-based hardmetals bonded with iron alloys: a review
}

\begin{abstract}
Growing concerns over the use of cobalt as binder for WC-based hardmetals has directed research efforts towards finding a suitable alternative binder offering comparable or even superior properties than those found in $\mathrm{WC}-\mathrm{Co}$ hardmetals. Complete substitution of cobalt by iron alloys has been extensively explored in several studies with significant improvements in mechanical properties of WC bonded with Fe alloys when carbon content addition is strictly controlled in powder composition. Asides from the commonly studied hardness and fracture toughness properties, transverse rupture strength property of this composites has also been observed to hold future promise with further development in the microstructural parameters such as porosity during sintering. This article reviews the progress in the mechanical properties of $\mathrm{WC}-\mathrm{Fe}$ alloys hardmetals.
\end{abstract}

Keyword: Cemented carbides; Eta phase; Mechanical properties; Wear resistance; Carbon content 